\title{
ON A FACTORISATION OF \\ POSITIVE DEFINITE MATRICES
}

Kulendra N. Majindar

(received November 2, 1962)

All our matrices are square with real elements. The Schur product of two $n \times n$ matrices $B=\left(b_{i j}\right)$ and $C=\left(c_{i j}\right)$ $(i, j,=1,2, \ldots, n)$, is an $n \times n$ matrix $A=\left(a_{i j}\right)$ with $a_{i j}=b_{i j} c_{i j},(i, j=1,2, \ldots, n)$.

A result due to Schur [1] states that if $B$ and $C$ are symmetric positive definite matrices then so is their Schur product A. A question now arises. Can any symmetric positive definite matrix be expressed as a Schur product of two symmetric positive definite matrices? The answer is in the affirmative as we show in the following theorem.

THEOREM. A real symmetric positive definite matrix is a Schur product of two real symmetric positive definite matrices.

Proof. Let us first prove the theorem for a real symmetric positive definite matrix $A_{1}=\left(a_{i j}^{\prime}\right)$ where $a_{i i}=1$, $(i=1,2, \ldots, n)$. For each $i, \quad(i=1,2, \ldots, n)$, let the $i$ characteristic roots of the leading $i \times i$ principal submatrix of $A_{1}$ (i. e. the submatrix occupying the upper left hand corner of $A_{1}$ ) be $\lambda_{1 i}, \lambda_{2 i}, \ldots, \lambda_{i i}$ with $\lambda_{1 i} \geq \lambda_{2 i} \geq \ldots \geq \lambda_{i i}>0$. Write $\lambda=\min \lambda_{i i}$. Choose $\alpha$ to be a positive number i

satisfying $1-\lambda<\alpha<1$. If $B$ is an $n \times n$ matrix with 1 in its main diagonal and $\alpha$ elsewhere, and $C=\left(c_{i j}\right)$ where $c_{i j}=a_{i j}^{1} / \alpha, i \neq j, \quad c_{i i}=1, \quad(i, j=1,2, \ldots, n)$, then the Schur

Canad. Math. BuIl. vol.6. no. 3, September 10,63 
product of $\mathrm{B}_{1}$ and $\mathrm{C}$ is $\mathrm{A}_{1}$.

Now we have to show that the matrices $B_{1}$ and $C$ are positive definite. Indeed the $i-t h$ leading principal minor is easily seen ( $\operatorname{see}[2])$ to be equal to $(1-\alpha)^{i-1}(1+i \alpha-\alpha), \quad(i=1,2, \ldots, n)$. So $B_{1}$ is positive definite. With regard to $C$, let us consider the following polynomial of degree $i$ in $x$ :

$$
P_{i}(x)=\left|\begin{array}{ccccc}
x & a_{12}^{\prime} & a_{13}^{\prime} & \ldots & a_{1 i}^{\prime} \\
a_{21}^{\prime} & x & a_{23}^{\prime} & \ldots & a_{2 i}^{\prime} \\
\ldots & \ldots & \ldots & \ldots & \ldots \\
a_{i 1}^{\prime} & a_{i 2}^{\prime} & a_{i 3}^{\prime} & \ldots & x
\end{array}\right|
$$

According to the definition of $\lambda_{1 i}, \lambda_{2 i}, \ldots, \lambda_{i i}$, we have $P_{i}\left(1-\lambda_{1 i}\right)=P_{i}\left(1-\lambda_{2 i}\right)=\ldots=P_{i}\left(1-\lambda_{i i}\right)=0$, and $P_{i}(x)>0$ whenever $x>1-\lambda_{i i}$. The leading principal minor of order $i$ of $C$ is equal to $\alpha^{-i} \mathrm{P}_{i}(\alpha)$. As $\alpha>0$ and $\alpha>1-\lambda_{i i}$, we see that this leading principal minor is positive. This holds for $i=1,2, \ldots, n$. Thus $C$ is a symmetric positive definite matrix. Hence we have got a desired factorisation of $\mathrm{A}$.

Let now $A=\left(a_{i j}\right),(i, j=1,2, \ldots, n)$, be a symmetric positive definite matrix in which not all the main diagonal elements are 1 . We know that $a_{i i}>0$ for $i=1,2, \ldots, n$. Put $a_{i j}^{\prime}=a_{i j} /\left(a_{i i} a_{j j}\right)^{\frac{1}{2}},(i, j=1,2, \ldots, n)$. Then $A_{1}=\left(a_{i j}^{\prime}\right)$ is a symmetric positive definite matrix, because the leading $i-t h$ principal minor of $A_{1}$ is equal to the product of $\left(a_{11} a_{22} a_{33} \cdots a_{i i}\right)^{-1}$ and the leading $i-t h$ principal minor of $A$, and so is positive, $(i=1,2, \ldots, n)$. Further, we define $B=\left(b_{i j}\right)$ where $b_{i i}=a_{i ;}$ and $b_{i j}=\alpha\left(a_{i i} a_{j j}\right)^{\frac{1}{2}}$ if $i \neq j$. 
Then $B$ is symmetric and positive definite, since its $i-t h$ principal minor is equal to the product of $\left(a_{11} a_{22} \cdots a_{i i}\right)$ and the leading $i-$ th principal minor of $B_{1}$, i.e. equal to $a_{11} \cdots a_{i i}(1-\alpha)^{i-1}(1+i \alpha-\alpha)$.

We now see that $A$ is the Schur product of the symmetric positive definite matrices $B$ and $C$, with $\alpha$ and $a_{i j}^{\prime}$ as defined above. This completes the proof.

We notice that each real symmetric positive definite matrix can be exhibited as a Schur product of two real symmetric positive definite matrices in infinitely many ways. Combining the above result with Schur's result, we can state that a real symmetric matrix is positive definite if and only if it is the Schur product of two real symmetric positive definite matrices.

\section{REFERENCES}

1. Richard Bellman, Introduction to Matrix Analysis, McGraw Hill, 1960, p. 94.

2. F.E. Hohn, Elementary Matrix Algebra, MacMillan Company, 1958, p. 50.

Loyola College, Montreal 